\title{
Fault Diagnosis for Transmission Lines Systems Using ANFIS Techniques
}

\author{
Azriyenni Azhari Zakri \\ Department of Electrical Engineering \\ Faculty of Engineering \\ Universitas Riau, Indonesia
}

Azhari

Zakri,

azriyenni@eng.unri.ac.id

\begin{abstract}
This paper presents a fault diagnosis for long transmission lines using Adaptive Neuro-Fuzzy Inference System (ANFIS). The electric power transmission system is a link power generation and distribution. If a failure occurs as long the transmission line could be estimation caused of undesired fault power delivery to consumer come not go well. Therefore, it would need to provide an alternative solution to solve this problem. The objectives of this paper are classification and estimate of a fault into the transmission line by using application of ANFIS. The systems have been put forward and tested on simulated data transmission lines into different faults. The results test given to contribute to an alternate technique where it has good performance for fault diagnosis in the transmission lines.
\end{abstract}

KEYWORDS: ANFIS, Classification, Estimate, Fault, Transmission Lines.

\section{INTRODUCTION}

The fault that occurs in the transmission line expect to be avoided, utility problems and equipment damage. It will overcome like arc and so on etc. These failures have disrupted the reliability of the operation of the power system. Different researchers will come in this problem that it suggested various schemes and algorithms. There are several techniques to detect failures in the transmission system, which is: time domain, frequency domain, and wavelet transform and hybrid intelligent technique $[1,2]$.

L. de Andrade et al. (2014) presented a review of the state of the art for fault location methods traveling waves. A critical analysis in transmission lines based on showing the advantages and disadvantages of each of them and their most common applications. A comparative analysis of two of the most widely used methods is carried out using real faults [3].

Sunil Singh and D. N. Vishwakarma (2015) presented survey and review of research the transmission lines. Transmission lines are an integral part of the power system and its main aim is to transmit the generated power to the consumer with the least interruption. A comprehensive review of various algorithms that have been developed and used over the last few years for the detection and classification of faults in transmission lines and mainly concentrate on following algorithm and their implementation for fault analysis such as, wavelet transform (WT), discrete wavelet transform (DWT), multiresolution analysis (MRA), wavelet energy entropy, artificial neural network (ANN), wavelet neuro-fuzzy approach and support vector machine (SVM) [4].

Mahdi Raoofat et al. (2016) presented the traveling waves method of fault location in transmission lines. The method is based on analysis of reflected from fault point. The received signal is processed by wavelet transform, and signal characteristics are used as input for the neural network. After training a neural network, the algorithm estimates the location of fault with reasonable accuracy. The algorithm is independent of the network configuration or length of the line and is trained once for each voltage level [5].

This paper describes the design and development of new techniques it can classify and estimate of type by using a Hybrid Intelligent Techniques. It is also introduced the name of ANFIS techniques. This type of faults is simulated one line to ground fault, two lines to ground fault, two-line fault, and three line fault. The results of a study are expected good ability of the method that has been used previously.

\section{TRANSMISSION LINE SYSTEM}

In this paper, Neuro-Fuzzy or familiar with the name of an Adaptive Neural Fuzzy Inference System (ANFIS). ANFIS is present using Fuzzy If-Then Rules into Neural Network construction [6].

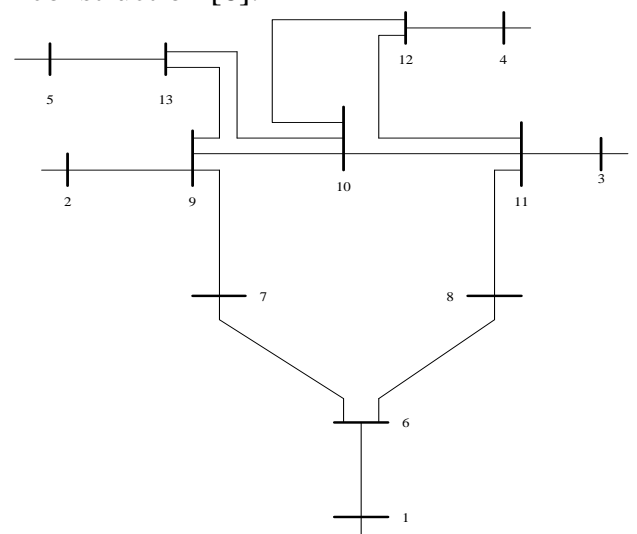

Figure 1: Transmission Lines 13 bus systems 
They are used appropriate learning and reduce the outcome of fault based on the data of training set. Fig. 1 shows the transmission line 13 bus systems $400 \mathrm{kV}$. ANFIS techniques offer a technique for Fuzzy modeling to study the output of the data set. It is obtained parameters of the membership functions associated with the fuzzy inference system to tread input or output of data [7]. The transmission systems are modeled with 13 bus systems would be shown in Fig. 2 to generate classification faults. The parameters are used data that have been generated by Matlab/Simulink.

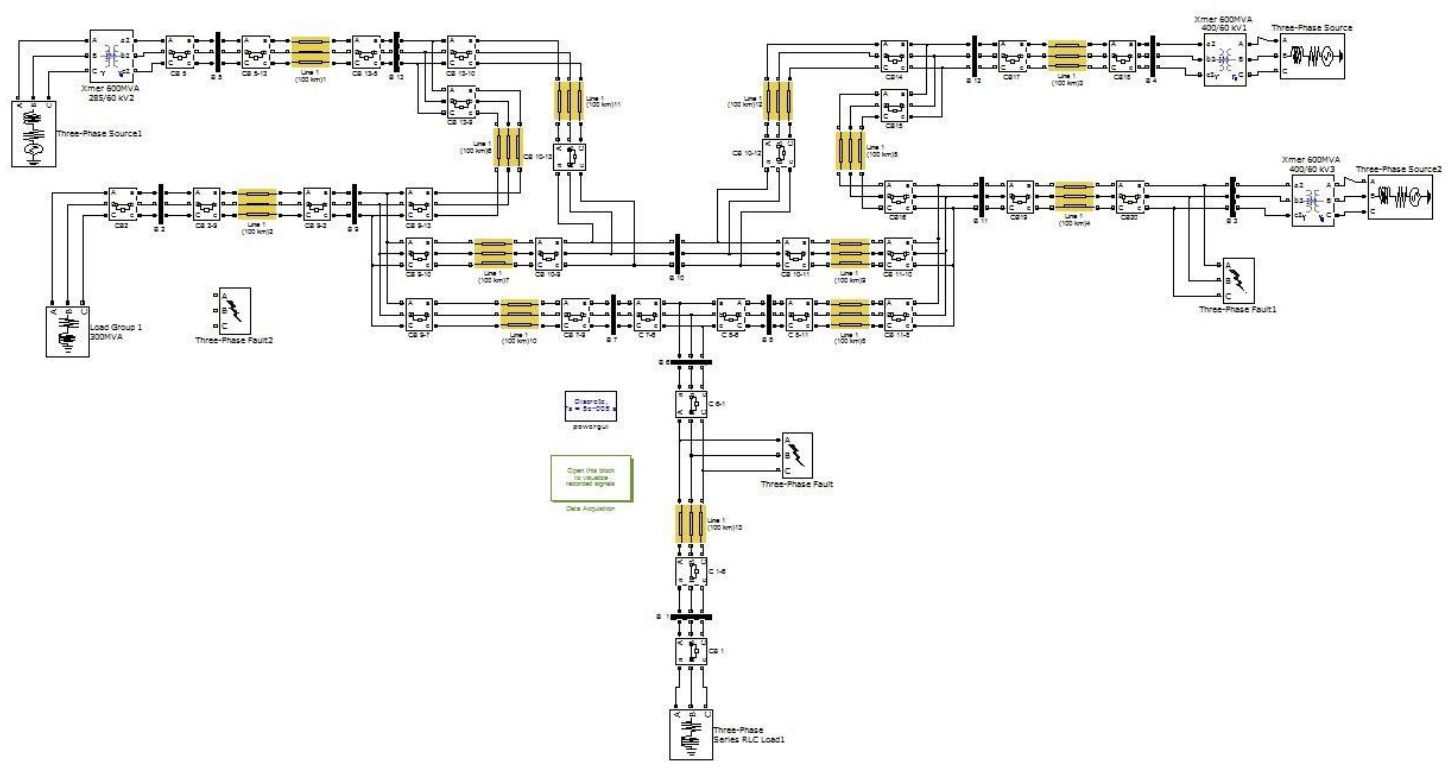

Figure 2: Transmission Lines 13 bus systems 400 kV via Simulink

\section{RESEARCH METHODOLOGY}

A Fuzzy System is composed of five stages, namely; Fuzzy Sets, Fuzzifiers, Fuzzy Rules, Inference Engine, and Defuzzifiers. Fuzzy Inference System is applied to the modeling of the system which is not clear. This system has a structure of rules defined by used commentation of the features of the model variables. The membership functions are selected in some situations modeling and indistinguishable to the membership function. It is showed at the data [8].

Fuzzy Inference System (FIS) is a way of mapping a space input to output using by Fuzzy Logic. The FIS try to formulate the reasoning turn of human language by the method of Fuzzy system. The Membership functions are authorized as a graphically reflect the fuzzy system. The $\mathrm{x}-$ axis reflects the universe of discourse, while the y-axis reflects the membership degrees in the [0,1] interval [9].

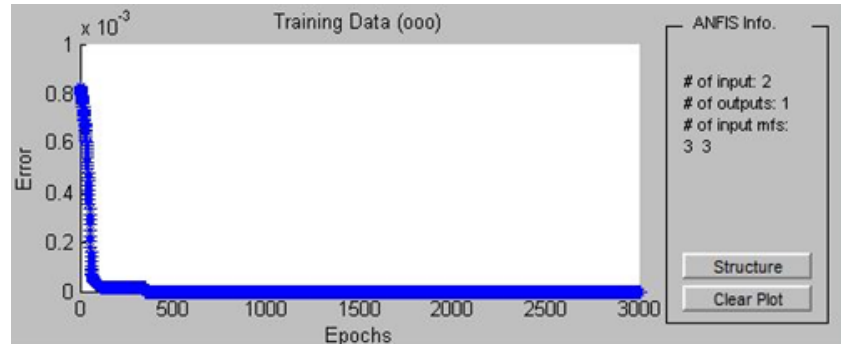

Figure 3: Training Data for ANFIS Techniques
Defuzzification for modify the fuzzy sets acquired by the inference engine into a crisp value. These parameters can be modified through the learning process. Fig. 3 shows training data fo ANFIS with 3000 iterations (epochs). Fig. 4 indicates the composition of the ANFIS consisting of inputs, fuzzification, inference, and defuzzification. ANFIS functions are a detector consisting of two neurons in the input layer as much as 3, and two Membership Function (MF) for each input as much as 6 and membership functions to the output constant $[7,10]$.

Layer 1: input.

Each node of this layer carries out membership degree, which is included to each of compatible fuzzy, sets by using membership functions.

$$
\begin{aligned}
& O_{1, i}=\mu_{A_{1}}(x) \text { for } i=1,2 \\
& O_{1, i}=\mu_{B_{i-2}}(y) \text { for } i=3,4
\end{aligned}
$$

Where: $x, y$ are crisp inputs to node $i$ and $A_{i}, B_{i}$ are the linguistic labels be coming membership functions $\mu_{A_{i}}, \mu_{B_{i}}$, in every each other.

The membership function can be any appropriate function that is continuously differentiable such as; Gaussian, trapezoidal, generalized bell and triangular [14]. The membership function in this paper shows in Fig. 2.

$$
\begin{aligned}
& \mu_{A_{i}}=\frac{1}{1+\left|\frac{x-c_{i}}{a_{i}}\right|^{2 b_{i}}} \\
& \mu_{B_{i-2}}=\frac{1}{1+\left|\frac{y-c_{i}}{a_{i}}\right|^{2 b_{i}}}
\end{aligned}
$$




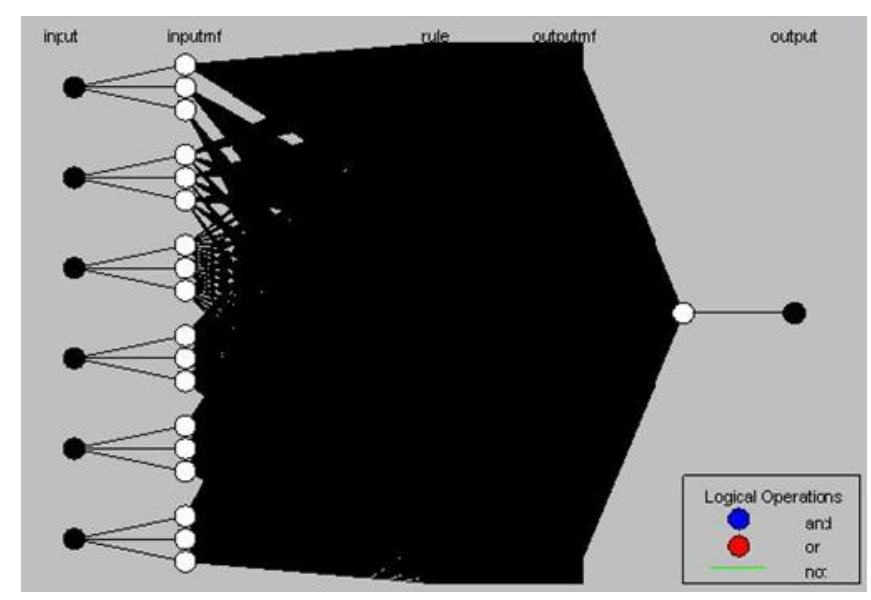

Figure 4: The Structure of ANFIS Techniques

Where: $\left\{a_{i}, b_{i}, c_{i}\right\}$ is parameter of the membership functions in the premise part of fuzzy if-then rules that changes the shapes of the membership function.

\section{Layer 2: fuzzy rules}

The AND operator used up to one output which indicates the result of the antecedent for a rule, i.e., firing strength. Firing strength means the degree antecedent part of a fuzzy rule, which satisfied, and it shapes the output function for the rule. On the other hand, the output $O_{2, k}$, of this layer are the products of appropriate degrees.

$$
O_{2, k}=w_{k}=\mu_{A_{i}}(x) * \mu_{B_{i}}(y)
$$

$i=1,2 ; \quad j=1,2 ; \quad k=2(i-1)+j$

Layer 3: average

The main objective is to enumerate the ratio of each rule's firing strength to the sum of all rules firing strength. In every each special chase, $\overline{w_{l}}$ is taken as the normalized strength.

$$
O_{3, i}=\overline{w_{l}}=\frac{w_{i}}{w_{1}+w_{2}+w_{3}+w_{4}} \quad i=1,2,3,4
$$

Layer 4: consequent

The node function of the fourth layer count up the effect of each rule toward the total output and establish it as follow;

$$
O_{3, i}=\overline{w_{l}} z_{i}=\overline{w_{l}}\left(p_{i} x+q_{i} y+r_{i}\right) \quad i=1, \ldots, 4
$$

Layer 5: output

The single node calculates all of the output by summing all the incoming signals. In consequence, the defuzzification creates process each rule's fuzzy results into a crisp output in this layer.

$$
O_{5,1}=\sum_{i=1}^{4} \bar{w}_{i} z_{i}=\frac{w_{1} z_{1}+w_{2} z_{2}+w_{3} z_{3}+w_{4} z_{4}}{w_{1}+w_{2}+w_{3}+w_{4}}
$$

ANFIS is more compound than the fuzzy inference system, and all the options are not ready for the fuzzy inference system. ANFIS is only appropriate for Sugeno type system. Also, have characteristics as follow:

- Be the first or zeroth order Sugeno type systems.

- Has one output which is acquired by using the weighted medium defuzzification. All output membership functions must be similar and be ribbed or constant.

- Amount of the output membership functions must be common to the rules number.

I have a unity weight for each rule.

\section{RESUlt AND Discussion}

Training data which is used to train ANFIS with a different fault. The condition for this type of fault; one line to ground, two lines to ground, two lines and three lines. Table 1 shows the data systems for the transmission line system. Also, Table 2 shows the data as short circuit faults (symmetry and non-symmetric) with different fault conditions, that is one line ground fault, two- line ground fault, two-line fault, and three line fault.

Table 1: Data System For Transmission Lines

\begin{tabular}{|l|l|}
\hline \multicolumn{1}{|c|}{ Data Systems } & \multicolumn{1}{c|}{ Values } \\
\hline Nominal Voltage & $400 \mathrm{kV}$ \\
\hline Frequency of System & $50 \mathrm{~Hz}$ \\
\hline Length of Transmission Lines & $100 \mathrm{~km}$ \\
\hline Impedance of Positive Sequences & $(9.79+j 110.23) \Omega$ \\
\hline The impedance of Negative Sequences & $(9.1879+j 134.66) \Omega$ \\
\hline Impedance of Zero Sequences & $(96.45+j 335.26) \Omega$ \\
\hline
\end{tabular}

ANFIS cannot admit all the customization options that allow primary fuzzy inference. Meaningful, it must use the membership functions and defuzzification functions that have been provided in the tools of ANFIS. Fig. 5 until Fig. 8 displays the results data show in the surface graph, where the inputs are voltage and current (Vabc/Iabc) of short circuit fault, respectively.

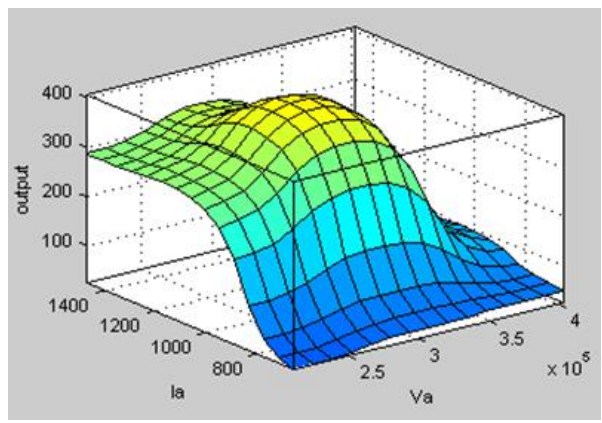

Figure. 5: Result in Surface Graph (Va/Ia) 
Table 2: Input Data with Different Fault For ANFIS

\begin{tabular}{|c|c|c|c|c|c|c|c|}
\hline \multicolumn{7}{|c|}{ Input ANFIS } & \multirow{2}{*}{ Target } \\
\hline Faults & $V_{a}(p u)$ & $V_{b}(p u)$ & $V_{c}(p u)$ & $I_{a}(p u)$ & $I_{b}(p u)$ & $I_{c}(p u)$ & \\
\hline \multirow{3}{*}{ ag } & 0.34 & 1.55 & 1.18 & 2.81 & 1.05 & 1.00 & 50 \\
\hline & 0.54 & 1.23 & 1.13 & 2.98 & 1.07 & 1.01 & 250 \\
\hline & 0.56 & 1.02 & 1.14 & 2.50 & 1.09 & 1.01 & 350 \\
\hline \multirow{3}{*}{ bcg } & 1.54 & 0.60 & 0.61 & 1.11 & 3.66 & 1.91 & 50 \\
\hline & 1.47 & 0.57 & 0.71 & 1.14 & 3.47 & 2.06 & 250 \\
\hline & 1.39 & 0.59 & 0.52 & 1.14 & 3.27 & 2.08 & 350 \\
\hline \multirow{3}{*}{$\mathrm{bc}$} & 1.00 & 0.73 & 0.75 & 1.00 & 3.65 & 2.89 & 50 \\
\hline & 1.00 & 0.70 & 0.64 & 1.00 & 3.55 & 2.84 & 250 \\
\hline & 1.00 & 0.51 & 0.52 & 1.00 & 3.34 & 2.90 & 350 \\
\hline \multirow{3}{*}{$a b c$} & 0.76 & 0.44 & 1.18 & 2.47 & 2.63 & 2.39 & 50 \\
\hline & 0.93 & 0.53 & 1.23 & 2.12 & 2.29 & 2.65 & 250 \\
\hline & 0.78 & 0.60 & 0.90 & 2.04 & 2.17 & 2.13 & 350 \\
\hline
\end{tabular}

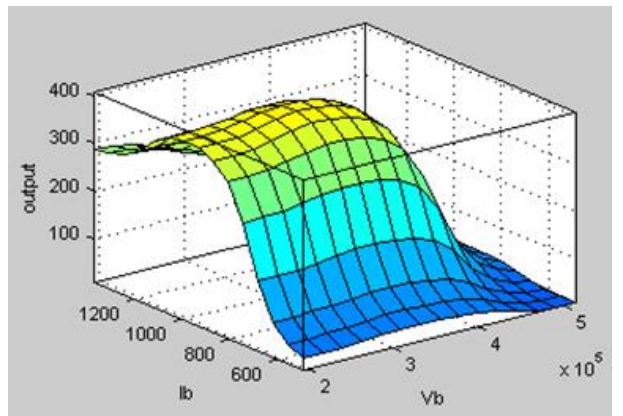

Figure. 6: Result in Surface Graph (Vb/Ib)

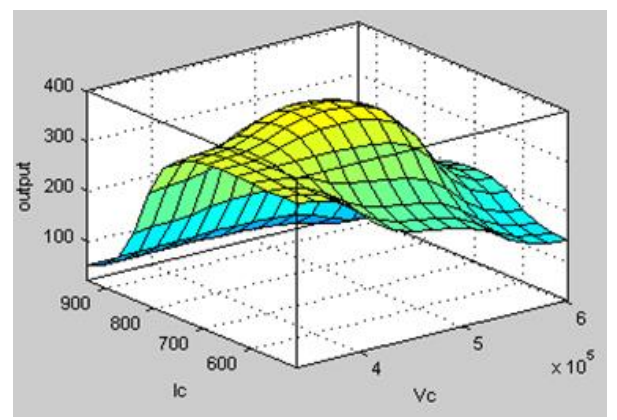

Figure. 7: Result in Surface Graph (Vc/Ic)

The results system simulation Matlab Simulink and Matlab GUI are shown in Tables 3 and 4. The input entered to the ANFIS then magnitude and angle of the three-phase currents and test data for the training process.
Table 3: Error of Fault Diagnosis

\begin{tabular}{|c|c|c|c|}
\hline \multirow{3}{*}{ Fault } & Target $(\mathbf{K m})$ & Output $(\mathbf{K m})$ & Error (\%) \\
\hline \multirow{4}{*}{ ag } & 50 & 50.00 & $0.0001 \%$ \\
\cline { 2 - 4 } & 250 & 250.00 & $0.0007 \%$ \\
\cline { 2 - 4 } & 350 & 350.00 & $0.0006 \%$ \\
\hline \multirow{3}{*}{ abg } & 50 & 50.00 & $0.0000 \%$ \\
\cline { 2 - 4 } & 250 & 249.99 & $0.0037 \%$ \\
\hline \multirow{3}{*}{ bc } & 350 & 350.00 & $0.0003 \%$ \\
\cline { 2 - 4 } & 50 & 50.00 & $0.0001 \%$ \\
\cline { 2 - 4 } & 250 & 250.01 & $0.0036 \%$ \\
\hline \multirow{3}{*}{ abc } & 350 & 349.99 & $0.0060 \%$ \\
\cline { 2 - 4 } & 50 & 50.00 & $0.0001 \%$ \\
\cline { 2 - 4 } & 250 & 250.00 & $0.0009 \%$ \\
\hline \multirow{3}{*}{} & 350 & 349.99 & $0.0031 \%$ \\
\hline
\end{tabular}

All values obtained from the corresponding fault was given as input for the ANFIS techniques. The result is better to deliver to values as supposed. These outputs are values of the ANFIS.

The above options will reduce the expected fault. After that, in Tabel 3 shows the error values of the proposed techniques for fault diagnosis.

The simulation results are:

- $\quad$ Number of nodes: 1503

- $\quad$ Number of linear parameters: 729

- $\quad$ Number of nonlinear parameters: 54

- Total number of parameters: 783 
- $\quad$ Number of training data pairs: 12

- $\quad$ Number of fuzzy rules: 729

\section{CONCLUSION}

ANFIS has served in this paper which is a combination of Fuzzy with the Neural Network. Then, Fuzzy system serves as the Fuzzy Inference System. The proposed ANFIS models are approach demonstrated successful performance for classification and estimation fault. Type of fault is presented one line to ground fault, two lines to ground fault, two-line fault, and three-line fault. All of the test data fault diagnosis has been done to get good output approach as the faulty classification of the test data provided relatively near perfect output. The demonstrated results fault diagnosis of the index percentage error of calculated against expected results is up to $1 \%$. Finally, test results can contribute to one of the intelligent alternative techniques that have very good performance in fault diagnosis transmission lines.

\section{REFERENCES}

[1] Aastha Aggarwal, H.M., Rajneesh Sharma, "Feature Extraction using EMD and Classification through Probabilistic Neural Network for Fault Diagnosis of Transmission Line," International Conference on Power Electronics. Intelligent Control and Energy Systems (ICPEICES), 2016.

[2] Fan YU, S.Z., Baolong LIU, "Based on the Wavelet Function of Power Network Fault Location," TELKOMNIKA. 11(4): p. 1924-1929, 2013.

[3] L. de Andrade, T.P.d.L., "Fault Location for Transmission Lines Using Wavelet," IEEE Latin America Transactions, 12(6), 2014
[4] Sunil Singh, D.N.V, "Intelligent Techniques for Fault Diagnosis in Transmission lines An Overview," International Conference on Recent Developments in Control, Automation and Power Engineering (RDCAPE), 2015.

[5] Mahdi Raoofat, A.M., Alireza Abunasri, "Fault Location in Transmission Lines Using Neural Network and Wavelet Transform," International Congress on Electric Industry Automation (ICEIA), Shiraz University, Iran. International Congress on Electric Industry Automation (ICEIA), Shiraz University. Iran.

[6] Reddy, M.J. and D.K. Mohanta, "Adaptive-neuro-fuzzy inference system approach for transmission line fault classification and location incorporating effects of power swings," IET Generation, Transmission \& Distribution, 2(2): p. 235, 2008.

[7] Yuanyuan Chai, L.J., and Zundong Zhang, "Mamdani Model-based Adaptive Neural Fuzzy Inference System and its Application," World Academy of Science, Engineering, and Technology 3 (27), 2009.

[8] Kevin Warwick, A.E.a.R.A., "Artificial Intelligence Techniques in Power Systems," IET Power and Energy series 22. London, United Kingdom: The Institution of Engineering and Technology, 2008.

[9] N. Sarikaya, K.G.a.C.Y., "Adaptive Neuro-Fuzzy Inference System For The Computation Of The Characteristic Impedance And Effective Permittivity Of The Micro Coplanar Strip Line," Progress In Electromagnetics Research B. p. 225-237, 2008.

[10] Zadeh, H.K, "Fuzzy-Neuro Approach to Investigating Transformer Inrush Current," 2006. 